\title{
Kirigami-processed cellulose nanofiber films for smart heat dissipation by convection
}

\author{
Kojiro Uetani $\mathbb{1}^{1}$, Keitaro Kasuya², Jiahao Wang ${ }^{2}$, Yintong Huang' ${ }^{1}$, Rikuya Watanabe ${ }^{3}$, Shota Tsuneyasu4', \\ Toshifumi Satoh ${ }^{3}$, Hirotaka Koga (1) ${ }^{1}$ and Masaya Nogi ${ }^{1}$
}

\begin{abstract}
Heat dissipation has become increasingly important in electronics. Conventional convection cooling systems have significant material and dimensional constraints, and they have difficulty meeting the heat dissipation, miniaturization, and flexibility requirements of next-generation smart electronics. Here, we used kirigami (the traditional art of paper cutting) with a thermally conductive cellulose nanofiber film to propose a flexible cooling system through convective heat dissipation. By stretching the Amikazari (net decoration) pattern produced by kirigami and allowing air convection through its aperture at $3.0 \mathrm{~m} / \mathrm{s}$, the thermal resistance was reduced to approximately one-fifth of that without kirigami and convection. The kirigami apertures defined the outlet air velocity, resulting in a significant increase in the heat-transfer coefficient. Our kirigami heat dissipation concept enables the design of electronics using a variety of film materials as shape-variant cooling structures, which will inspire a wide range of thermal engineering and electronics applications.
\end{abstract}

\section{Introduction}

Heat dissipation systems play an important role in preventing the thermal runaway of electronic devices with ever-increasing exhaust heat, ensuring normal operation and safety, and determining the design freedom of electronic devices. In particular, the next generation of thin and flexible electronics must provide smart applications, longevity, multifunctionality, flexibility, and wearability ${ }^{1}$, so it is essential to achieve these functions along with heat dissipation. Conventional cooling systems use convection to transfer the heat from a heat source to a convecting fluid. While this provides high cooling performance, it requires heat sinks with many metal/ceramic fins, as well as thermal interface materials ${ }^{2}$ to improve adhesion and thermal conduction with hard heat sources. Therefore, the components inevitably become bulky, making it

\footnotetext{
Correspondence: Kojiro Uetani (uetani@eco.sanken.osaka-u.ac.jp)

${ }^{1}$ SANKEN (The Institute of Scientific and Industrial Research), Osaka University, Mihogaoka 8-1, Ibaraki-shi, Osaka 567-0047, Japan

${ }^{2}$ Graduate School of Engineering, Osaka University, Mihogaoka 8-1, Ibaraki-shi, Osaka 567-0047, Japan

Full list of author information is available at the end of the article
}

difficult to fabricate thin and flexible electronic devices. Thermal design, especially for small heat-generating components, largely relies on thermal diffusion to the substrate $^{3}$. While a system that diffuses heat throughout the substrate and housing by thermal conduction is expected to make the entire electronic to be thinner and more flexible than when using heat sinks, it requires a large area and a composite material with high thermal conductivity to provide sufficient cooling ${ }^{4-7}$, which ultimately leads to larger components and less flexibility. A heat dissipation mechanism that maintains flexibility is needed for the development of next-generation highperformance multifunctional devices.

Paper electronics have recently been developed as flexible and functional devices based on cellulose nanofiber (CNF) film substrates, such as flexible nonvolatile memory ${ }^{8,9}$, paper antennas ${ }^{10}$, transistors ${ }^{11}$, conductive paper $^{12}$, and humidity sensors ${ }^{13}$. These applications originate from the high performance of CNF films, including toughness (mechanical strength of $100-500 \mathrm{MPa})^{14,15}$ and thermal stability (small coefficients of thermal expansion $)^{16}$. In particular, for bottom-emission type (i.e.,

\section{(c) The Author(s) 2021}

(c) (i) Open Access This article is licensed under a Creative Commons Attribution 4.0 International License, which permits use, sharing, adaptation, distribution and reproduction cc) in any medium or format, as long as you give appropriate credit to the original author(s) and the source, provide a link to the Creative Commons license, and indicate if changes were made. The images or other third party material in this article are included in the article's Creative Commons license, unless indicated otherwise in a credit line to the material. If material is not included in the article's Creative Commons license and your intended use is not permitted by statutory regulation or exceeds the permitted use, you will need to obtain permission directly from the copyright holder. To view a copy of this license, visit http://creativecommons.org/licenses/by/4.0/. 
substrate transmission type) light-emitting devices, such as powder electroluminescent (EL) devices, CNF film is the most preferred substrate material because it has higher transparency and smoothness than plastic films or pulp papers, providing high luminescence ${ }^{17}$. As higher performance is achieved in these devices, the heat dissipation problem will become more significant. Therefore, it may be useful to use ascidian tunicate-derived CNF (TCNF) films, which have recently been found to show inplane thermal conductivities as high as $2.5 \mathrm{~W} / \mathrm{mK}$, which is 3-10 times higher than those of polymer films (usually $<1 \mathrm{~W} / \mathrm{mK}$ ) often used as flexible substrates for electronics ${ }^{18,19}$. This is because even a small improvement in the thermal conductivity of the substrate material (in particular, polymeric materials with small thermal conductivity) has been estimated to significantly reduce the maximum temperature of a heat-generating light-emitting diode chip ${ }^{20}$. However, thermal diffusion cooling will not be able to keep up with the increase in heat generation.

To advance the further cooling of flexible twodimensional (2D) films, it is essential to cut a slit in the film and allow fluid to flow through to induce convection cooling. The regular fin structure found in conventional heat sinks can be formed in the film. The closest structure to that structure is the periodic void structure created by kirigami. Kirigami is the traditional art of cutting paper into a wide range of three-dimensional (3D) structures with dimensional transformation over time (i.e., fourdimensional (4D) structures) by making a number of periodic incisions, and it is used in various engineering applications ${ }^{21}$. The simple incised design called "Amikazari" (net decoration) is highly versatile, and it is used not only in everyday decorations such as Tanabata (the Star Festival) paper ornaments but also in solar-cell substrates with optical-axis tracking ${ }^{22}$, diffraction gratings with variable spacing ${ }^{23}$, programmable multistable configurations of hydrogels ${ }^{24}$, graphene kirigami ${ }^{25}$, stretchable lithium-ion batteries ${ }^{26}$, stretchable bioprobes ${ }^{27}$, wearable sensors $^{28}$, and strain sensors ${ }^{29}$. Unlike most of the above applications that focus on stretching dynamism and metamaterial designability for future 4D electronics, here, for the first time, we focus on the periodic void structure of kirigami that enhances interaction with air and forms an effective convection path. The kirigami structure allowing convection through the aperture is expected to be a novel smart heat dissipation platform. Elucidating the heat dissipation using kirigami-processed CNF film is thought to maximize the heat dissipation effect for general-purpose flexible film.

In this study, we verified the synergistic effect of convection and thermal conduction by kirigami. We clearly demonstrated the excellent cooling performance of a kirigami-processed CNF film through a heat dissipation test with a pseudo heat source using photothermal conversion by irradiating pseudo sunlight on a graphitesprayed blackened area. The contributions of convection and thermal conduction to the heat dissipation of the kirigami specimen were characterized. We also demonstrated the heat-dissipation effect of powder EL devices, whose high heat generation has been a problem in their use, by forming them onto a CNF film and processing them into a kirigami EL device.

\section{Results \\ Heat-dissipation test for kirigami}

Amikazari-patterned kirigami specimens were prepared by laser processing of T-CNF films (Fig. 1a). Preliminary experiments showed that the T-CNF film with abundant carboxyl groups introduced on the CNF surface showed extended burning along the processed lines, suggesting that cellulose degradation was in progress even outside the pattern line. In addition, the extended burning made it difficult to process patterns with fine incision intervals (in particular, $a=1 \mathrm{~mm}$ ) because the processed line was not precisely straight. Therefore, the introduction of carboxyl groups on the CNF surface by 2,2,6,6-tetramethylpiperidine-1-oxyl (TEMPO) oxidation was limited to a minimum amount to assist in fibrillation, resulting in the introduction of $0.204 \mathrm{mmol} / \mathrm{g}$ to prevent composition changes and improve the processability. As a result, kirigami specimens with incision intervals of $1-4 \mathrm{~mm}$ could be accurately cut without extended burning around the processed patterns, as shown in Fig. $1 \mathrm{~b}$.

A $1 \mathrm{~cm} \times 1 \mathrm{~cm}$ blackened area was formed by spraying graphite in the center of only one side of the specimen as a pseudo heat source to test the heat dissipation performance of the kirigami specimen (Fig. 1a). The specimen was held with the blackened surface facing the ground and irradiated with intense white light $\left(0.56 \mathrm{~W} / \mathrm{cm}^{2}\right)$ to generate heat by photothermal conversion ${ }^{30}$. The light irradiation intensity in this study was set based on the result that the powder EL device using a CNF film substrate showed a maximum current of $1.87 \mathrm{~mA}$ at $300 \mathrm{~V}^{17}$, which was calculated to be $0.56 \mathrm{~W} / \mathrm{cm}^{2}$ of heat generated by assuming that all of the energy was converted to heat. The graphite-sprayed blackened area showed an absorption rate of more than $90 \%$ over a wide wavelength range and absorbed the incident light well (Fig. S1), while the unblackened CNF film did not effectively absorb light to generate heat $^{30}$ (see Fig. S2). Because the film surface of the stretched kirigami specimen was inclined, a custommade jig was prepared to hold the film surface horizontally (Fig. 1c). This jig allowed the film to be tilted and stretched around the center of the film where the blackened area formed. The temperature distribution was observed from the top surface of the film using a thermography camera, and the maximum temperature was 


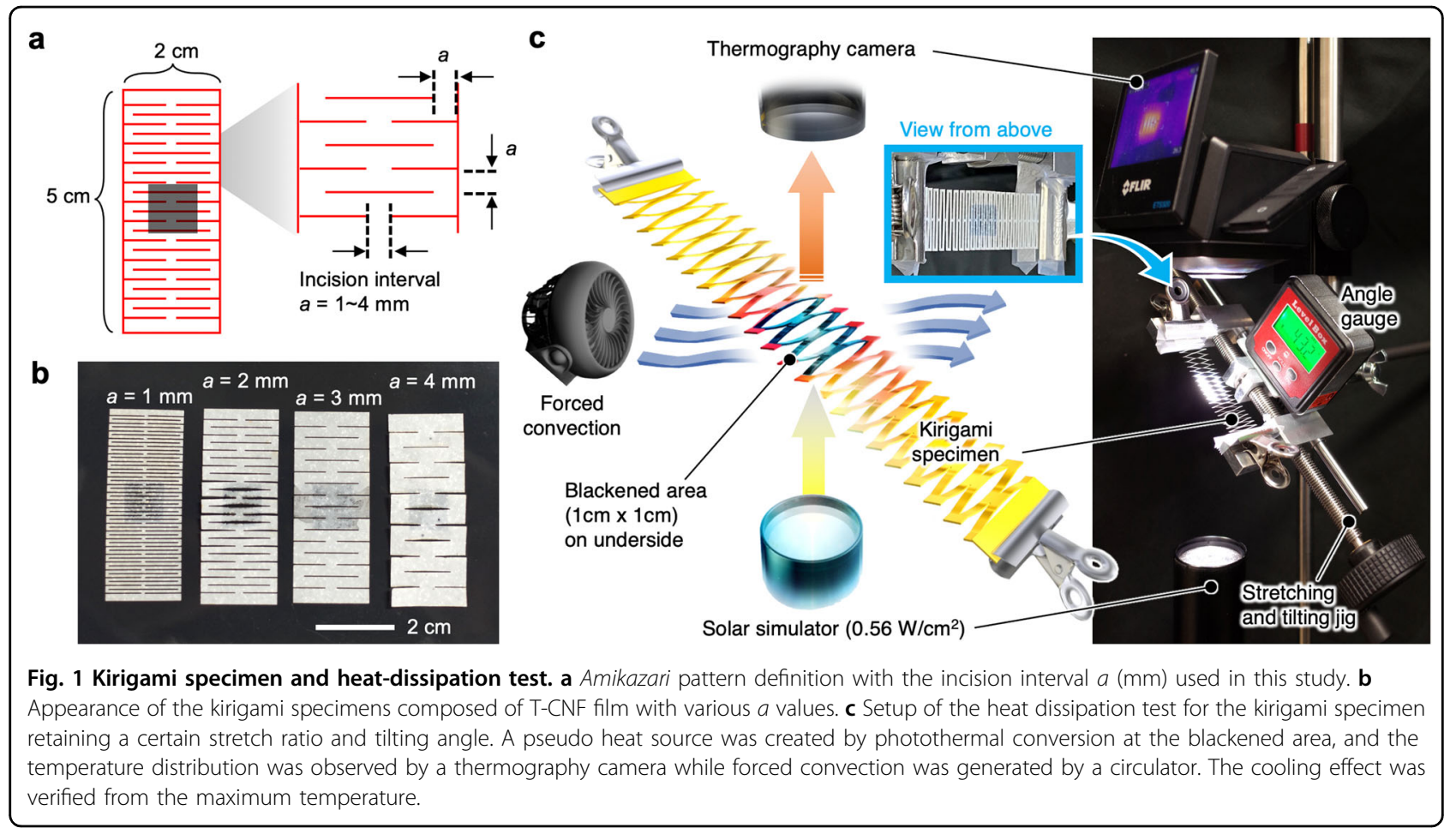

determined. The heat dissipation test was conducted using a circulator to generate forced convection from the horizontal direction.

\section{Convective heat dissipation by kirigami}

The stretch ratio of the kirigami specimen was calculated by $D_{\text {stretch }} / D_{0}$ (Fig. 2a). After determining the amount of stretching, the film surface was tilted so that it was horizontal, and forced convection was applied to the film surface. Preliminary experiments revealed that the inlet air velocity before passing through the kirigami specimen and the outlet air velocity after passing through the specimen were different even under the same test conditions. Therefore, we installed anemometer sensors at the inlet and outlet positions shown in Fig. $2 \mathrm{~b}$.

The maximum temperature of the T-CNF film before kirigami processing was $152 \pm 2.3^{\circ} \mathrm{C}$ during natural convection and $74.1 \pm 1.5^{\circ} \mathrm{C}$ during forced convection for an inlet air velocity of $3.0 \mathrm{~m} / \mathrm{s}$ (Fig. 2c, d). The corresponding thermal resistance $\left(R_{\mathrm{th}}\right)$ values during natural and forced convection were calculated to be 230 and $91.3 \mathrm{~K} / \mathrm{W}$, respectively, using the equation $R_{\mathrm{th}}=\Delta T / P$, where $\Delta T$ and $P$ are the temperature increment and heat input $(0.56 \mathrm{~W})^{31}$. The in-plane thermal conductivity of the T-CNF film was measured to be $2.2 \pm 0.2 \mathrm{~W} / \mathrm{mK}$, which is reasonable compared with previously reported values ${ }^{18,32}$. The heat from the blackened area propagated to the surrounding area for several $\mathrm{mm}$ (Fig. 2c, d). When the $a=2 \mathrm{~mm}$ kirigami specimen was stretched 1.43 times, the maximum temperature was $124 \pm 5.3^{\circ} \mathrm{C}\left(R_{\text {th }}=180 \mathrm{~K} / \mathrm{W}\right)$ during natural convection, but the specimen promptly cooled to $50.3 \pm 0.4{ }^{\circ} \mathrm{C}\left(R_{\mathrm{th}}=48.8 \mathrm{~K} / \mathrm{W}\right)$ by convection at $3.0 \mathrm{~m} / \mathrm{s}$ (Fig. 2e, f). The kirigami specimen reduced the thermal resistance to approximately one-fifth of that without kirigami and convection. The heat was efficiently transferred to the atmosphere in a steady manner, demonstrating the high convection-cooling performance of kirigami.

Interestingly, even though the inlet air velocity was always set to $3.0 \mathrm{~m} / \mathrm{s}$, the outlet air velocity was found to significantly vary depending on the stretch ratio of the kirigami specimen (Fig. 2g). The outlet air velocity at a stretch ratio of 1.43 asymptotically approached $3.0 \mathrm{~m} / \mathrm{s}$, which was almost the same as the inlet air velocity. The kirigami aperture seemed to show flow resistance, limiting the air velocity through the kirigami specimen. Therefore, the vertical aperture distance $b$, which is defined in Fig. $2 \mathrm{~b}$, was mathematically obtained for each stretch ratio. From the side view of the kirigami aperture shown in the upper left insert of Fig. $2 \mathrm{~h}$, the simple relationship

$$
b=2 a \tan \theta
$$

was established, where $\theta$ can be calculated from the basic relationship of $D_{\text {stretch }} / D_{0}=\cos ^{-1} \theta$ for the Amikazari pattern. As $b$ increased from 0 to $\sim 2 \mathrm{~mm}$, the outlet air 


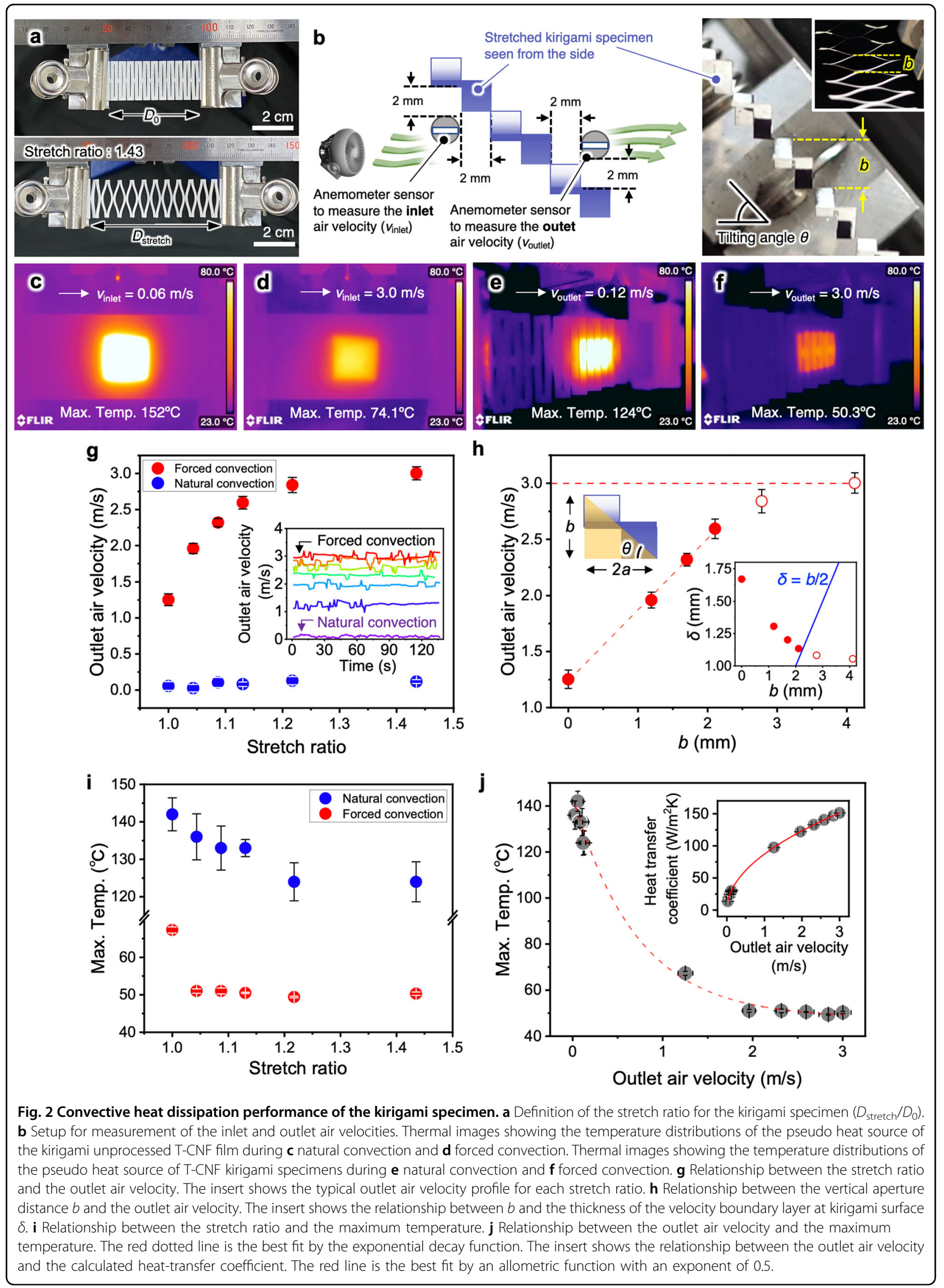


velocity linearly increased, and it gradually approached the inlet air velocity of $3.0 \mathrm{~m} / \mathrm{s}$ when $b$ was greater than $\sim 2 \mathrm{~mm}$ (Fig. 2h). We believe that this flow resistance is affected by the thickness of the velocity boundary layer at the kirigami surface $\delta$. We thus estimated $\delta$ using the equation $\delta \cong 5 x / \sqrt{R e}$, where $x$ is the distance from the tip of the flat plate (corresponding to $2 a$ in the case of our kirigami specimen $)^{33}$. $R e$ is the Reynolds number: $R e=\frac{v_{a} \cdot L}{\nu}$, with $\nu=\frac{\mu}{\rho}, \mu=\frac{1.4592 \cdot T_{\mathrm{f}}^{\frac{3}{2}}}{109.10+T_{\mathrm{f}}} \times 10^{-6}$, and $\rho=\frac{351.99}{T_{\mathrm{f}}}+$ $\frac{344.84}{T_{\mathrm{f}}^{2}}$, where $v_{a}$ is the air velocity, $v$ is the kinematic viscosity of air, $\mu$ is the viscosity of air, $\rho$ is the density of air and $T_{\mathrm{f}}$ is the film temperature, which is the average temperature of the heat source and the air ${ }^{31,34}$. Because the velocity boundary layer occurs on both sides of the kirigami specimen, the relationship between the approximate $\delta$ and $b / 2$ is plotted in the lower right insert of Fig. 2h. $\delta$ was then found to be larger than $b / 2$ when $b$ was 0 to $\sim 2 \mathrm{~mm}$. This result fully corresponds to the fact that the outlet air velocity is constrained by $b$ when $b$ is less than $\sim 2 \mathrm{~mm}$ (Fig. 2h). The aperture distance owing to stretching the kirigami specimen limits the outlet air velocity if it is within two times the velocity boundary layer thickness.

For both natural and forced convection, the maximum temperature decreased as the stretch ratio increased (Fig. 2i). In particular, the maximum temperature of the unstretched kirigami specimen was comparable with that of the T-CNF specimen without kirigami processing (Fig. $2 \mathrm{c}$ and $\mathrm{d}$ ), indicating that the 3D structural change caused by kirigami significantly contributed to convective heat dissipation. We demonstrated that the maximum temperature exponentially decayed with the outlet air velocity (Fig. 2j). This heat dissipation performance owing to convection can be expressed by the heat transfer coefficient, which represents the transferability of heat from the heat source to the convection air. The local heat transfer coefficient $h\left(\mathrm{~W} / \mathrm{m}^{2} \mathrm{~K}\right)$ was calculated by

$$
h=\frac{\lambda \cdot N u}{L}
$$

where $\lambda, N u$, and $L$ are the thermal conductivity of air, Nusselt number, and representative length, respectively ${ }^{3}$. $L$ of the kirigami specimen was defined as the specimen length through which the convecting air passes, that is, the incision interval $a$. According to a previous study ${ }^{34}, \lambda$ for air was calculated by $\lambda=\frac{2.3340 \times 10^{-3} \cdot T_{\mathrm{f}}^{\frac{3}{2}}}{164.54+T_{\mathrm{f}}}$. We used the maximum temperature of each specimen measured by thermography for the heat-source temperature. When forced convection was applied, laminar flow was assumed based on the small $R e$ values of 143 to 360 for stretch ratios of 1 to 1.43 (well within the range of $<5 \times 10^{5}$ for laminar flow $)^{33}$. Nu was calculated by $N u=0.664 \cdot R e^{\frac{1}{2}}$. $\operatorname{Pr}^{\frac{1}{3}}$. $P r$ is the Prandtl number: $\operatorname{Pr}=\frac{\mu \cdot C}{\lambda}$, where the specific heat of air $C=1030.5-0.19975 \cdot T_{\mathrm{f}}+3.9734 \times 10^{-4}$. $T_{\mathrm{f}}^{231,34}$.

In the case of natural convection, when assuming a downward heating plate, $\mathrm{Nu}$ was calculated by the equation $\mathrm{Nu}=0.27 \cdot(\mathrm{Gr} \cdot \mathrm{Pr})^{\frac{1}{4}}$, where $\mathrm{Gr}$ is the Grashof number ${ }^{31}$. However, in the case of the kirigami specimens with small $L$ values $(1-4 \mathrm{~mm})$, the values of $\mathrm{Gr} \operatorname{Pr}$ were found to be very small $(\sim 40)$, and they greatly deviated from the range in which natural convection can be assumed $\left(10^{5}<\operatorname{Gr} \operatorname{Pr}<10^{10}\right)^{31,33}$. This indicates that the buoyancy is not sufficient to be considered natural convection. When $L$ is small, the area around the heating plate is not thermally stable, and the buoyancy caused by the temperature difference is unlikely to be a clear convection-driving source. Therefore, even at low air velocities with small $R e$ values of 2-13 without applying any forced convection, it can be regarded as equivalent to laminar flow in forced convection because of its extremely low buoyancy owing to the small $a$ value of the kirigami specimen. In this study, laminar flow, as in forced convection, was assumed for the natural-convection air velocity in the kirigami specimen to calculate the heattransfer coefficient.

The heat-transfer coefficient of the kirigami specimen under natural convection at $0.12 \mathrm{~m} / \mathrm{s}$ (Fig. 2e) was calculated to be $29.6 \mathrm{~W} / \mathrm{m}^{2} \mathrm{~K}$, while that under forced convection at $3.0 \mathrm{~m} / \mathrm{s}$ was $151.0 \mathrm{~W} / \mathrm{m}^{2} \mathrm{~K}$, an increase of about five times. From the above equation, the heat-transfer coefficient is proportional to the 0.5th power of the air velocity, so fitting with an allometric function with an exponent of 0.5 gave a good approximation for the relationship between the heat-transfer coefficient and the outlet air velocity (insert of Fig. 2j). We clearly demonstrated that the convective heat-dissipation performance of kirigami specimens with small $L$ values can be expressed using the outlet air velocity, which is the velocity of air passing through the kirigami specimen, and that thermal design can be achieved by the heat-transfer coefficient based on the forced-convection model, even in natural-convection mode.

Although the thermal resistance for convection heat transfer $R_{\text {conv }}\left(=(h \cdot A)^{-1}\right.$, where $A$ is the heat-transfer area $^{31}$ is often used to describe the heat-dissipation performance of heat sinks and their surfaces ${ }^{35}$, the $A$ value of the kirigami specimen (i.e., the blackened area) has a unique feature of almost constant $A$ against the structural change by stretching, unlike heat sinks. We discussed the heat dissipation performance using only the heat-transfer coefficient, which more directly describes kirigami cooling. Similarly, the thermal resistance owing to thermal radiation was considered to be equal because the surface 
area and emissivity of the kirigami specimens made of T-CNF film were constant regardless of the stretch ratio.

When the kirigami specimens with $a=1,2,3$, and $4 \mathrm{~mm}$ were stretched at a stretch ratio of 1.22 , the heat dissipation performance was highest for $a=1 \mathrm{~mm}$, and it was almost the same for $a=3$ and $4 \mathrm{~mm}$ (Fig. S3a). For the same stretch ratio, a larger $a$ makes $b$ larger, which should be advantageous for heat dissipation. However, the outlet air velocity was almost the same at $2.6-2.8 \mathrm{~m} / \mathrm{s}$ for all of the specimens with $a=1-4 \mathrm{~mm}$ (the insert in Fig. $\mathrm{S} 3 \mathrm{~b})$. At a stretching ratio of 1.22 , any $a$ might have given $b$ values that exceeded double $\delta$. Therefore, the reason for the change in heat dissipation (different maximum temperatures) depending on $a$ in Fig. S3a is thought to be that the longer $a$ is, the less heat is transported to the film edge, and the amount of heat dissipated per unit area (i.e., the heat transfer coefficient) decreases. The calculated heat-transfer coefficient showed the same exponential decay trend (Fig. S3b) as in Fig. 2i. We speculate that the heat dissipation performance of kirigami film is determined by the balance between the outlet air velocity, which is defined by the $a$-value and the stretching ratio, and the thermal conductivity of the film.

In addition, there was no significant difference in heat dissipation when the film thickness was changed from 37 to $94 \mu \mathrm{m}$ (Fig. S3c). Although the CNF film has a large anisotropy of thermal conductivity between the in-plane and thickness directions ${ }^{18}$, the heat would have been sufficiently transferred in the thickness direction during spreading in the plane direction because the heat propagates in the in-plane direction over a distance of a few $\mathrm{mm}$, which is much larger than that of $\sim 50 \mu \mathrm{m}$ in the thickness direction. Considering that the heat dissipation is strongly influenced by the incision interval (Fig. S3a), we believe that the in-plane thermal conduction of the kirigami film mainly determined the heat dissipation in the steady state.

In the case of a conventional heat sink with a constant envelope volume, two factors largely compete to determine the heat dissipation performance: the fin spacing and the surface area. As the fin spacing increases, the air velocity between the fins increases and the heat dissipation performance improves, but as the surface area decreases, the heat dissipation performance reaches a head $^{31}$. As a result, the optimal heat dissipation performance is determined by the balance between the air velocity between the fins and the surface area. Conversely, unlike heat sinks, the kirigami specimen can be assessed using a single factor, the outlet air velocity, which enables a simpler heat-dissipation design. In addition, our kirigami films can use various air directions to dissipate heat depending on their design. As shown in Fig. S4, if we design a propeller-shaped pattern by kirigami, we can use a perpendicular air direction for heat dissipation instead of a parallel air direction as in the Amikazari pattern.

\section{Conductive heat dissipation by kirigami}

Another factor that affects the heat dissipation of kirigami is the thermal conductivity of the film. We processed Amikazari patterns with $a=2 \mathrm{~mm}$ using film materials with thermal conductivities that greatly vary from $\sim 1$ to $400 \mathrm{~W} / \mathrm{mK}$ (Fig. 3a) and conducted similar heat dissipation tests. An in-plane thermal conductivity of $0.9 \mathrm{~W} / \mathrm{mK}$ has been reported for the polyimide film ${ }^{18}$, and literature values of $16,90.5,237$, and $398 \mathrm{~W} / \mathrm{mK}$ were used for stainless steel (SUS-304), $\mathrm{Ni}, \mathrm{Al}$, and $\mathrm{Cu}$ foils, respectively ${ }^{33}$. The differences among the thermal conductivities of the materials were particularly pronounced during natural convection, and the heat spread was significantly different, as shown in the thermal images in Fig. $3 \mathrm{a}$. To accurately measure the temperature of the metallic materials, the entire surface of the thermographic side was blackened with graphite spray to reduce the infrared reflection and enhance the emissivity (see Fig. S2). We also analyzed the surface roughness of all the films using a scanning probe microscope (SPM), as shown in Fig. S5. The root-mean-square roughness $(\mathrm{Rq})$ of the $\mathrm{T}$-CNF film at the $20 \mu \mathrm{m}$ square was slightly larger than that of PI and metal films due to fiber accumulation. However, the Rq of $\sim 27 \mathrm{~nm}$ for the T-CNF film is almost equally small as that of $\sim 26 \mathrm{~nm}$ for the Al film, and it can be concluded that the irregularities might not affect the thickness of the velocity boundary layer.

The trend of the outlet air velocity was almost the same even when the film material was changed (Fig. 3b), and it reproduced a feature derived from the kirigami structure. This means that the heat-transfer coefficients determined by conditions such as the outlet air velocity and maximum temperature at each stretching ratio are equal regardless of the material. However, even though the air velocity was the same for the different materials, the maximum temperatures were significantly different, as shown in Fig. 3c. In both natural and forced convection, the maximum temperature was lower for higher thermal conductivity of the film material. At the same time, the temperatures of all of the materials decreased with increasing stretch ratio, which suggests that the effect of convection heat dissipation is similar to that in Fig. 2.

A clear trend was observed where the thermal conductivity of the film defined the maximum temperature for both natural and forced convection (Fig. 3d). T-CNF film, which has a relatively high thermal conductivity of $2.2 \mathrm{~W} /$ $\mathrm{mK}$ among polymeric films, also contributes to heat dissipation through thermal conduction. The result that films with higher thermal conductivity showed better cooling performance also occurs in conventional heat sinks. More importantly, polymeric films with low thermal conductivity, which could not previously be used as heat-dissipating components, also exhibited heat-dissipation performance at a practical level $\left(\sim 50^{\circ} \mathrm{C}\right)$ for a generated heat of $0.56 \mathrm{~W}$ in 


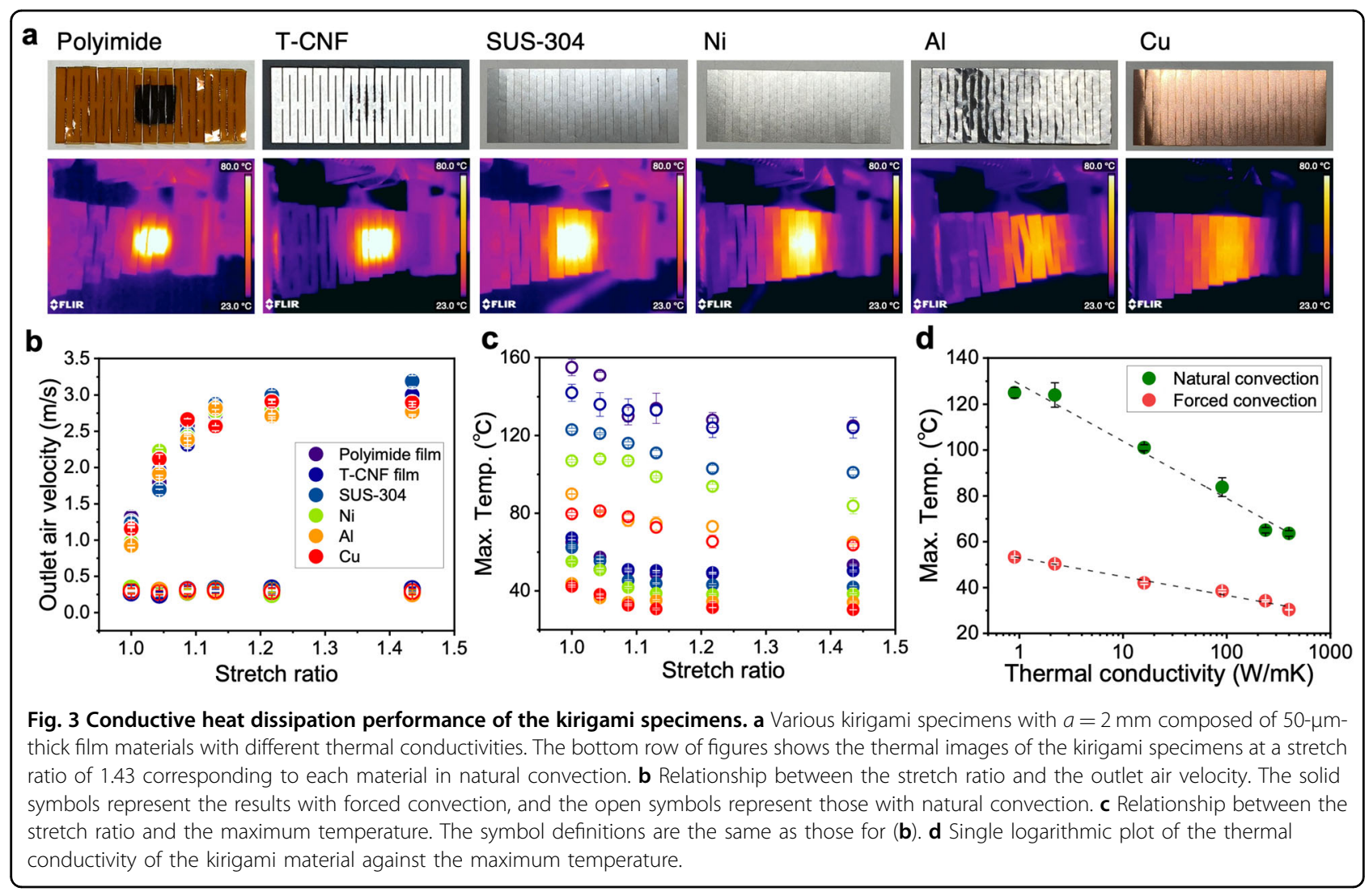

the kirigami specimens. It is difficult to use electrically conductive and opaque film materials, such as metal foils, as electronics substrates without additional processing irrespective of their thermal conductivity, while insulating and translucent polymeric films cannot dissipate heat because of their low thermal conductivity. The kirigami heat dissipation framework overcomes this material dilemma, and we have demonstrated that even polymer films with low thermal conductivity can achieve heat dissipation that can withstand practical use.

\section{Kirigami of EL devices}

To highlight the functionality of kirigami in addition to its heat dissipation, we fabricated a traditional bottomemission-type powder EL device on a T-CNF film (Fig. 4a). The powder EL device is a flexible flat light source that applies an alternating current (AC) voltage to zincsulfide-type phosphor particles printed on a film substrate $^{36}$. This device enables the development of smart, energy-saving, and multifunctional light sources, such as large-area display textiles ${ }^{37}$, stretchable electronics ${ }^{38,39}$, and deformable displays ${ }^{40}$, owing to its high environmental resistance and lack of need for sealing. However, there are concerns that the inevitably generated heat will rapidly decrease the dielectric constant of barium titanate at above its Curie temperature ${ }^{41,42}$, causing a significant decrease in the luminance and luminous efficiency because the set electric field cannot be applied, thus shortening the lifetime by EL degradation ${ }^{43}$ and allowing glass transition for conventional polymeric packages. Therefore, the development of an effective cooling mechanism to enhance the luminous efficiency (i.e., promote energy savings), extend the lifetime, and improve the safety of use has remained an issue.

Our EL device with an area of $\sim 6 \mathrm{~cm} \times 5 \mathrm{~cm}$ printed on the T-CNF film emitted light through the translucent T-CNF substrate at an AC effective voltage of $165 \mathrm{~V}$ at $1.2 \mathrm{kHz}$ to show EL spectra comparable with those previously reported ${ }^{17}$ (Fig. S6). This powder EL device generated heat during the emission of light, reaching a maximum temperature of $73.5^{\circ} \mathrm{C}$ (Fig. 4b). We then manually cut a double-column Amikazari pattern with $a$ $=2.5 \mathrm{~mm}$ into the $3.5 \mathrm{~cm} \times 5 \mathrm{~cm}$ area of this EL. After making the cut, the device continued to emit light while expanding and contracting because both of the electrodes still percolated (Fig. 4c). At a stretch ratio of $\sim 1.3$ times, the maximum temperature of the device was $68.6^{\circ} \mathrm{C}$ under natural convection, but it rapidly cooled to $34.7^{\circ} \mathrm{C}$ when exposed to convection at $3.0 \mathrm{~m} / \mathrm{s}$ while emitting light (Fig. 4d, e). By lowering the temperature of the powder EL to the same level as body temperature, it is possible to make the device wearable (Fig. 4f). Convective 

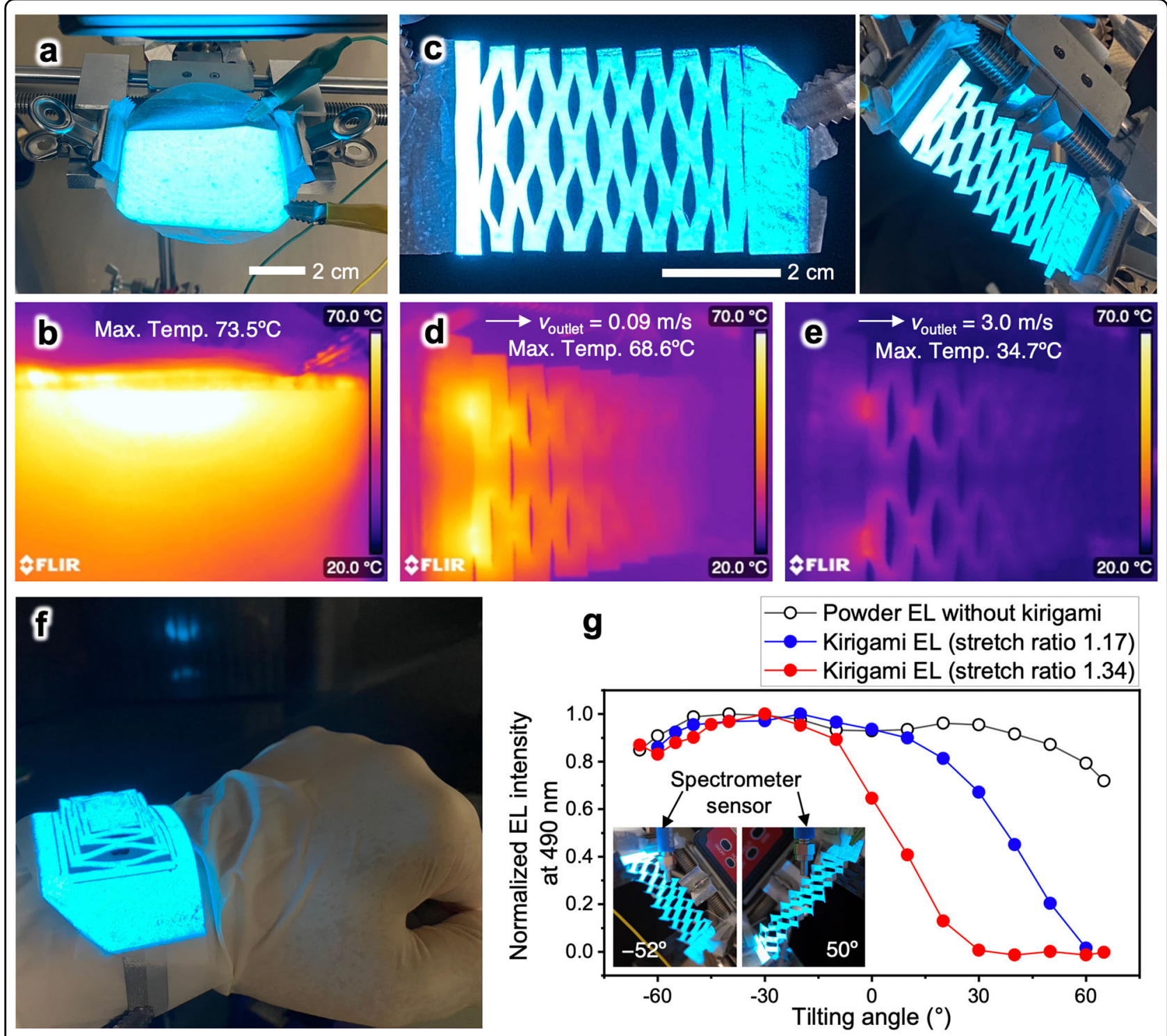

Fig. 4 Kirigami EL device. a Powder EL device formed on the T-CNF film and $\mathbf{b}$ its thermal image during emission. (c) Kirigami-processed EL device. Thermal images of the emitting kirigami EL device under $\mathbf{d}$ natural and $\mathbf{e}$ forced convection at a stretch ratio of $~ 1.3$. $\mathbf{f}$ Demonstration of a wearable kirigami EL device. $\mathbf{g}$ Maximum emission intensity of each EL spectrum plotted against the tilting angle.

heat dissipation by kirigami processing and the T-CNF film package realized a sufficiently cool powder EL device while maintaining flexibility for the first time.

Another functionality of kirigami is the controllability of the emission-angle range. Originally, the powder EL device showed almost no optical-axis directivity, but when it was stretched, the light-emitting surface was inclined, and thus, the emission angle could be dynamically modulated. By detecting the luminescence intensity at a tilt angle of $\pm 60^{\circ}$ to the stretching direction using a spectrometer, we showed an emission-angle range that clearly differed depending on the stretching magnification (Fig. 4g). The theoretically calculated tilting angles were $31.3^{\circ}$ and $41.7^{\circ}$ for stretch ratios of 1.17 and 1.34 , respectively. This slight difference in the tilting angle was found to restrict the light emission angle. We demonstrated that kirigami provides good controllability of the emission-angle range with the stretch ratio for ELs without optical-axis directionality.

\section{Discussion}

We have demonstrated a kirigami heat-dissipation system that allows air convection through the periodic apertures of the Amikazari pattern. The kirigami aperture size determined by the stretch ratio defined the outlet air velocity and significantly enhanced the heat-transfer 
coefficient. Although the thermal conductivity of the film itself is as important as that of a conventional heat sink, we demonstrated that even a polymer-based film with low thermal conductivity can be made to function as a heatdissipating component by using kirigami structures. Kirigami of the powder EL device cools it to approximately the same temperature as the human body, which is sufficiently cool to make it wearable, and allows the emission-angle range to be actively controlled by the stretch ratio. The variety of kirigami patterns, which are not limited to the Amikazari pattern, will make it possible to design dramatically more flexible heat-dissipation structures at will, as well as to improve the energy efficiency, extend the service life and improve the safety of electronics. Our heat dissipation concept based on kirigami processing of film materials will open up new pathways for the use of various conventional film materials as shape-variant cooling structures to freely design electronics.

\section{Methods}

\section{Preparation of CNF film}

The mantle of tunicate ascidian (Halocynthia roretzi) was cut into $1-\mathrm{cm}$ squares with scissors and soaked overnight in $3 \mathrm{wt} \% \mathrm{NaOH}$ solution for deproteinization. After washing, the product was bleached in $1 \mathrm{wt} \%$ $\mathrm{NaClO}_{2}$ solution at $80-90^{\circ} \mathrm{C}$ under acidic conditions for $4 \mathrm{~h}$ until the product became white. The product was then washed with distilled water to obtain tunicate cellulose pulp. The tunicate cellulose pulp ( $1 \mathrm{~g}$ dry weight) was dispersed in $100 \mathrm{~mL}$ of distilled water with the addition of $0.016 \mathrm{~g}$ of TEMPO and $0.1 \mathrm{~g}$ of $\mathrm{NaBr}$. Oxidation was initiated by adding $0.56 \mathrm{~mL}$ of $1.8 \mathrm{M} \mathrm{NaClO}$ aqueous solution, and the $\mathrm{pH}$ was kept higher than 10 by adding $0.5 \mathrm{M} \mathrm{NaOH}$. After reacting for $1.5 \mathrm{~h}$, the product was washed until the $\mathrm{pH}$ reached $\sim 7$ to obtain TEMPOoxidized tunicate pulp. The pulp suspension at $0.2-0.5 \mathrm{wt} \%$ was then agitated by a high-speed blender for $20 \mathrm{~min}$ for fibrillation. After filtering the unfibrillated residual pulp using a metal mesh (50 mesh), tunicate cellulose nanofibers (T-CNFs) were obtained. The T-CNF suspension was filtered with a membrane filter (A010A090C, Advantec, Tokyo, Japan), and another membrane filter was used to cover the surface of the wet mat. The sandwiched mat was then hot-pressed at $110^{\circ} \mathrm{C}$ and $\sim 1 \mathrm{MPa}$ for $10 \mathrm{~min}$ to dry the T-CNF film with a thickness of $46 \mu \mathrm{m}$. The in-plane thermal conductivity was calculated by the multiplication of the in-plane thermal diffusivity measured by a TA33 thermowave analyzer (Bethel Co., Ltd., Ibaraki, Japan), the heat capacity measured by a differential scanning calorimeter (Thermo Plus Evo2 8230, Rigaku, Tokyo, Japan) and the bulk density.

\section{Kirigami specimen preparation}

Amikazari patterns with incision intervals (a) of $1-4 \mathrm{~mm}$ were designed in a $5 \mathrm{~cm} \times 2 \mathrm{~cm}$ rectangle. The T-CNF film was processed using a $\mathrm{CO}_{2}$ laser processing machine (HAJIME CL1 Plus, Oh-Laser Co., Ltd., Saitama, Japan) at a cutting speed of $20 \mathrm{~mm} / \mathrm{s}$ and a laser intensity of 7.2 W. Polyimide film (Kapton 200H, Du Pont-Toray Co., Ltd., Tokyo, Japan) with a thickness of $50 \mu \mathrm{m}$ was similarly processed. However, it was not cut under the same conditions of laser processing because it only burned, so the patterned film was manually cut after processing. Metal foils (stainless steel (SUS-304), Ni, Al, and $\mathrm{Cu}$ ) with thicknesses of $50 \mu \mathrm{m}$ were purchased from Nilaco Corp. (Tokyo, Japan). Kirigami processing of the metal foil was outsourced to Takumi Precise Metal Work Manufacturing Ltd. (Osaka, Japan), and it was performed by a laser micromachining system (Nissha HC600, Nippon Sharyo, Ltd., Nagoya, Japan). The SUS-304, Ni and Al foils were processed at laser-processing speeds of 3000 , 3000 , and $1500 \mathrm{~mm} / \mathrm{min}$ with laser powers of 60,60 , and $530 \mathrm{~W}$, respectively, using $\mathrm{N}_{2}$ assist gas. The $\mathrm{Cu}$ foil was processed at a speed of $3000 \mathrm{~mm} / \mathrm{min}$ with a $400 \mathrm{~W}$ laser using $\mathrm{O}_{2}$ assist gas. The surface roughness (root-meansquare height, Rq) of each film was measured by a scanning probe microscope (SPM, AFM5000/AFM5300E, Hitachi High-Tech Corp., Tokyo, Japan) under cyclic contact mode using a cantilever (OMCL-AC160TS, Olympus Corp., Tokyo, Japan) with a spring constant of $k=26 \mathrm{~N} / \mathrm{m}$.

\section{Heat-dissipation test}

The heat dissipation test was conducted in a room environment with a temperature of $23 \pm 1{ }^{\circ} \mathrm{C}$ and relative humidity of $30 \% \pm 10 \%$. The blackened area was formed on the kirigami-processed CNF specimen by masking a $1 \mathrm{~cm} \times 1 \mathrm{~cm}$ area and coating it with graphite spray (FC-142, Fine Chemical Japan, Co., Ltd., Tokyo, Japan). Light absorption of the blackened area was measured by a UV-3600 Plus spectrophotometer (Shimadzu Corp., Kyoto, Japan) using an integrating sphere attachment (ISR-603, Shimadzu Corp.). A stretching and tilting jig was custommade to maintain the inclined posture of the kirigami specimen while stretching it a certain ratio. White light with an irradiance of $0.56 \mathrm{~W} / \mathrm{cm}^{2}$ was irradiated using a solar simulator (HAL-320W, Asahi Spectra Co., Ltd., Tokyo, Japan). The temperature distributions of the kirigami specimens were observed with a thermography camera (ETS320, FLIR Systems, Inc., Wilsonville, OR, USA). After waiting for a few minutes for the measurement environment to reach a steady state, 5-9 observations were made at intervals of several tens of seconds, and the median of the maximum temperatures was used. Forced convection was generated by a circulator (KJ-D994, Twinbird Corp. Niigata, Japan) placed at a distance of $60 \mathrm{~cm}$ from the 
center of the kirigami specimen. The air velocity was measured by a hot-wire anemometer (DT-8880, CEM, Shenzhen Everbest Machinery Industry Co., Ltd., Shenzhen, China). The air velocities for both natural and forced convection were measured by installing anemometer sensors in the horizontal direction. The average air velocity was calculated from the results of continuous measurements taken at $1 \mathrm{~Hz}$ intervals for $\sim 2$ min for each measurement condition.

\section{Fabrication and characterization of the powder EL device}

The powder EL device with a bottom-emission structure was fabricated by a previously reported procedure ${ }^{44}$. First, a high-dielectric polymer paste was prepared by mixing cyanoethyl polyvinyl alcohol (cyanoresin, CR-V, Shin-Etsu Chemical Co., Ltd., Tokyo, Japan) in cyclohexanone (037-05096, Fujifilm Wako Pure Chemical Co., Osaka, Japan) at a weight ratio of 3:7. This polymer paste was laminated on the T-CNF film by an automatic screenprinting machine (TU2020-C, Seritech Co., Ltd., Osaka, Japan) and dried at $80^{\circ} \mathrm{C}$ for $6 \mathrm{~min}$. Next, poly(2,3-dihydrothieno-1,4-dioxin)-poly(styrenesulfonate) (PEDOT: PSS, 768650, Sigma-Aldrich Co., LLC., Tokyo, Japan) was laminated and then dried at $80^{\circ} \mathrm{C}$ for $10 \mathrm{~min}$. Zinc sulfide phosphor particles (GG45, Osram Sylvania, Wilmington, MA, USA) were then dispersed in the high-dielectric polymer paste at a weight ratio of 4:6. This functional paste was laminated on the transparent electrode and dried at $80^{\circ} \mathrm{C}$ for $6 \mathrm{~min}$. $\mathrm{BaTiO}_{3}$ (620-07545, Kishida Chemical Co., Ltd., Osaka, Japan) was then dispersed in the high-dielectric polymer paste at a weight ratio of 4:6, laminated on the phosphor layer, and dried at $80^{\circ} \mathrm{C}$ for 6 min. Finally, silver paste (MP-603S, Mino Group Co., Ltd., Gifu, Japan) was laminated on the dielectric layer and dried at $80^{\circ} \mathrm{C}$ for $6 \mathrm{~min}$. A TP-HBA3-INV AC inverter with $165 \mathrm{~V}$ at $1.2 \mathrm{kHz}$ for the Flex-O-Lite Tracer Plate (TP-A3, Oriental Direct, Tokyo, Japan) was used to emit the EL. The optical fiber sensor of the spectrometer (SEC2020, ALS Co., Ltd., Tokyo, Japan) was held at a distance of $1 \mathrm{~cm}$ from the stretching axis of the EL device to measure the tilting angle dependence of the spectral intensity. The voltage dependence of the current and the luminance of the kirigami EL device were measured with an EL measurement system (SX-1152, Iwatsu Electric Co., Ltd., Tokyo, Japan).

\section{Acknowledgements}

We thank Mr. Yuki Matsushita of the mechanical workshop of SANKEN for production and adjustment of the stretching and tilting jig. We thank Mr. Masato Sasa of Takumi Precise Metal Work Manufacturing Ltd. for his kind cooperation in kirigami processing of the metal foils. We thank the Co-creation Bureau, Osaka University, for the DSC measurements. We thank Dr. Akira Kitajima of the Nanotechnology Open Facilities in Osaka University for his kind cooperation in SPM measurements. This work was supported by the Nanotechnology Platform of MEXT, Grant Number JPMXP09S210S0034. This research was partly supported by JSPS KAKENHI (Grant No. 21K14215 to S.T.) of the Japan Society for the Promotion of Science (JSPS), and the Fuji Seal Foundation. This work was performed under the Cooperative Research Program of "Network Joint Research Center for Materials and Devices". We thank Tim Cooper, PhD, from the Edanz Group (https://jp.edanz.com/ac) for editing a draft of this manuscript.

\section{Author details}

${ }^{1}$ SANKEN (The Institute of Scientific and Industrial Research), Osaka University, Mihogaoka 8-1, Ibaraki-shi, Osaka 567-0047, Japan. ${ }^{2}$ Graduate School of Engineering, Osaka University, Mihogaoka 8-1, Ibaraki-shi, Osaka 567-0047, Japan. ${ }^{3}$ Department of Media Engineering, Graduate School of Engineering, Tokyo Polytechnic University, 1583 liyama, Atsugi, Kanagawa 243-0297, Japan. ${ }^{4}$ Department of Electrical and Electronic Engineering, National Institute of Technology, Oita College, 1666 Maki, Oita 870-0152, Japan

\section{Author contributions}

K.U., S.T., H.K., T.S., and M.N. wrote the manuscript. K.U. and S.T. made the designs and conducted the experiments with the assistance of K.K., J.W., Y.H., R. W., and H.K. K.K. developed the materials and analyzed the data with the assistance of J.W. and K.U. Y.H. characterized the optical spectra. R.W., S.T., and T. S. fabricated and characterized the EL devices in cooperation with K.K. and K.U. T.S., H.K., and M.N. supervised the project.

\section{Competing interests}

The authors declare no competing interests.

\section{Publisher's note}

Springer Nature remains neutral with regard to jurisdictional claims in published maps and institutional affiliations.

Supplementary information The online version contains supplementary material available at https://doi.org/10.1038/s41427-021-00329-5.

Received: 12 May 2021 Revised: 4 August 2021 Accepted: 16 August 2021. Published online: 24 September 2021

\section{References}

1. Liu, Y., He, K., Chen, G., Leow, W. R. \& Chen, X. Nature-inspired structural materials for flexible electronic devices. Chem. Rev. 117, 12893-12941 (2017).

2. Uetani, $\mathrm{K}$. et al. Elastomeric thermal interface materials with high throughplane thermal conductivity from carbon fiber fillers vertically aligned by electrostatic flocking. Adv. Mater. 26, 5857-5862 (2014).

3. Kunimine, N. Erekutoronikusu no tameno Netsu-sekkei Kanzen Seiha (Complete Thermal Design for Electronics) (Nikkan Kogyo Shimbun, Ltd., 2018).

4. Wang, K., Li, M., Zhang, J. \& Lu, H. Polyacrylonitrile coupled graphite oxide film with improved heat dissipation ability. Carbon 144, 249-258 (2019).

5. $\mathrm{Ci}, \mathrm{H}$. et al. Enhancement of heat dissipation in ultraviolet light-emitting diodes by a vertically oriented graphene nanowall buffer layer. Adv. Mater. 31, 1901624 (2019).

6. Li, X. et al. Graphene heat dissipation film for thermal management of hot spot in electronic device. J. Mater. Sci.: Mater. Electron. 27, 7715-7721 (2016).

7. Hong, S., Yoo, S. S. \& Yoo, P. J. Binder-free heat dissipation films assembled with reduced graphene oxide and alumina nanoparticles for simultaneous high in-plane and cross-plane thermal conductivities. J. Mater. Chem. C. 7, 9380-9388 (2019).

8. Nagashima, K. et al. Cellulose nanofiber paper as an ultra flexible nonvolatile memory. Sci. Rep. 4, 5532 (2014).

9. Celano, U. et al. All-nanocellulose nonvolatile resistive memory. NPG Asia Mater. 8, e310 (2016).

10. Inui, T., Koga, H., Nogi, M., Komoda, N. \& Suganuma, K. A miniaturized flexible antenna printed on a high dielectric constant nanopaper composite. Adv. Mater. 27, 1112-1116 (2015).

11. Fujisaki, Y. et al. Transparent nanopaper-based flexible organic thin-film transistor array. Adv. Funct. Mater. 24, 1657-1663 (2014).

12. Koga, $\mathbf{H}$. et al. Uniformly connected conductive networks on cellulose nanofiber paper for transparent paper electronics. NPG Asia Mater. 6, e93 (2014). 
13. Kasuga, T., Yagyu, H., Uetani, K., Koga, H. \& Nogi, M. "Return to the soil" nanopaper sensor device for hyperdense sensor networks. ACS Appl. Mater. Interfaces 11, 43488-43493 (2019).

14. Henriksson, M., Berglund, L. A., Isaksson, P., Lindstrom, T. \& Nishino, T. Cellulose nanopaper structures of high toughness. Biomacromolecules 9 1579-1585 (2008)

15. Nakagaito, A. N., Iwamoto, S. \& Yano, H. Bacterial cellulose: the ultimate nanoscalar cellulose morphology for the production of high-strength composites. Appl. Phys. A 80, 93-97 (2005).

16. Diaz, J. A., Wu, X., Martini, A., Youngblood, J. P. \& Moon, R. J. Thermal expansion of self-organized and shear-oriented cellulose nanocrystal films. Biomacromolecules 14, 2900-2908 (2013).

17. Tsuneyasu, S. et al. Enhancement of luminance in powder electroluminescent devices by substrates of smooth and transparent cellulose nanofiber films. Nanomaterials 11, 697 (2021).

18. Uetani, K., Okada, T. \& Oyama, H. T. Crystallite size effect on thermal conductive properties of nonwoven nanocellulose sheets. Biomacromolecules 16 2220-2227 (2015)

19. Uetani, K., Okada, T. \& Oyama, H. T. Thermally conductive and optically transparent flexible films with surface-exposed nanocellulose skeletons. J. Mater. Chem. C. 4, 9697-9703 (2016).

20. Kunimine, N. Thermal management of high power LED system. J. Illum. Engng. Inst. Jpn 96, 799-802 (2012).

21. $\mathrm{Xu}, \mathrm{L}$., Shyu, T. C. \& Kotov, N. A. Origami and kirigami nanocomposites. ACS Nano 11, 7587-7599 (2017).

22. Lamoureux, A., Lee, K., Shlian, M., Forrest, S. R. \& Shtein, M. Dynamic kirigami structures for integrated solar tracking. Nat. Commun. 6, 8092 (2015).

23. $\mathrm{Xu}$, L. et al. Kirigami nanocomposites as wide-angle diffraction gratings. ACS Nano 10, 6156-6162 (2016).

24. Hao, X. P. et al. Kirigami-design-enabled hydrogel multimorphs with application as a multistate switch. Adv. Mater. 32, 2000781 (2020).

25. Blees, M. K. et al. Graphene kirigami. Nature 524, 204-207 (2015)

26. Song, Z. et al. Kirigami-based stretchable lithium-ion batteries. Sci. Rep. 5, 10988 (2015).

27. Morikawa, Y. et al. Ultrastretchable kirigami bioprobes. Adv. Healthc. Mater. 7 1701100 (2018).

28. Yamagishi, K. et al. Elastic kirigami patch for electromyographic analysis of the palm muscle during baseball pitching. NPG Asia Mater. 11, 80 (2019).

29. Baldwin, A. \& Meng, E. Kirigami strain sensors microfabricated from thin-film parylene C. J. Microelectromech. Syst. 27, 1082-1088 (2018).
30. Huang, Y., Morishita, Y., Uetani, K., Nogi, M. \& Koga, H. Cellulose paper support with dual-layered nano-microstructures for enhanced plasmonic photothermal heating and solar vapor generation. Nanoscale Adv. 2, 2339-2346 (2020).

31. Fukagawa, S. Hi-toshinku to Fan niyoru Netsusekkei no Kiso to Jissen (Basics and Practice of Thermal Design with Heat Sinks and Fans) (CQ Publishing Co., Ltd., 2019).

32. Adachi, $\mathrm{K}$. et al. Thermal conduction through individual cellulose nanofibers. Appl. Phys. Lett. 118, 053701 (2021).

33. The Japan Society of Mechanical Engineer. Heat Transfer (The Japan Society of Mechanical Engineers, 2005).

34. McQuillan, F. J., Culham, J. R. \& Yovanovich, M. M. Properties of Dried Air at One Atmosphere. UW/MHTL 8406, G-8401 (Microelectronics Heat Transfer Lab, University of Waterloo, 1984).

35. Bae, J. J. et al. Heat dissipation of transparent graphene defoggers. Adv. Funct. Mater. 22, 4819-4826 (2012).

36. Takeda, N. et al. Enhanced electroluminescence in fully printable powder electroluminescent device with flexible invisible silver-grid transparent electrode. Electron. Lett. 56, 612-614 (2020).

37. Shi, $\mathbf{X}$. et al. Large-area display textiles integrated with functional systems. Nature 591, 240-245 (2021).

38. Cho, S. et al. Highly stretchable sound-in-display electronics based on straininsensitive metallic nanonetworks. Adv. Sci. 8, 2001647 (2021).

39. Tan, Y. J. et al. A transparent, self-healing and high-K dielectric for low-fieldemission stretchable optoelectronics. Nat. Mater. 19, 182-188 (2020).

40. Yang, Z., Wang, W., Pan, J. \& Ye, C. Alternating current electroluminescent devices with inorganic phosphors for deformable displays. Cell Rep. Phys. Sci. 1, 100213 (2020).

41. Petrovsky, V., Petrovsky, T., Kamlapurkar, S. \& Dogan, F. Dielectric constant of barium titanate powders near curie temperature. J. Am. Ceram. Soc. 91 3590-3592 (2008).

42. Roberts, S. Dielectric and piezoelectric properties of barium titanate. Phys. Rev. 71, 890-895 (1947)

43. Chen, F., Kitai, A. H. \& Xiang, Y. Temperature-dependent degradation of AC powder EL. J. Electrochem. Soc. 156, H585-H587 (2009).

44. Takeda, N., Tsuneyasu, S. \& Satoh, T. EL emission enhancement in powder electroluminescent device via insertion of receptive layer. Electron. Lett. $\mathbf{5 6}$ 144-147 (2020). 